\title{
Vorschläge für eine auf die Bedürfnisse der Patienten ausgerichtete Mengensteuerung
}

Matthias Bäuml

9.1 Motivation für die Notwendigkeit einer Mengensteuerung - 170

9.2 Aktuelle Instrumente zur Mengensteuerung in Deutschland - 171

9.3 Instrumente für eine effektivere Mengensteuerung - 174

9.3.1 Reduktionen der Informationsasymmetrie durch Zweitmeinungsverfahren - 174

9.3.2 Reduktionen ungewollter finanzieller Anreize durch systemische Erneuerung der Methode zur Kategorisierung von Fallgruppen - 177

9.3.3 Reduktionen ungewollter finanzieller Anreize durch systemische Erneuerung der Methode zur Berechnung der Relativgewichte - 178

$9.4 \quad$ Fazit -181

Literatur - 182 


\section{- Zusammenfassung}

Patienten können die Wirkung einer vorgeschlagenen medizinischen Behandlung durch einen Leistungserbringer oftmals nicht genau einordnen, sodass Leistungserbringer über die Bedürfnisse der Patienten hinaus medizinische Behandlungen anbieten können. Eine zukunftsorientierte Mengensteuerung sollte vor diesem Hintergrund sicherstellen, dass sich die beobachtete Art und Menge von medizinischen Behandlungen so genau wie möglich an den tatsächlichen Bedürfnissen der Patienten orientieren. Die Instrumente der aktuellen Mengensteuerung können dieses Ziel nicht erreichen. Eine Weiterentwicklung der Zweitmeinung kann die Informationsasymmetrie zwischen Leistungserbringern und Patienten effektiv reduzieren. Zusätzlich kann eine Weiterentwicklung der Kategorisierung der Fallgruppen bzw. der Berechnung der Relativgewichte ungewollte finanzielle Anreize für die Leistungserbringer deutlich reduzieren. Für eine informierte und zukunftsorientierte Mengensteuerung ist es außerdem wichtig, dass der Gesetzgeber auch in Deutschland sicherstellt, dass die relevanten patientenbezogenen Informationen mit einer zeitlich adäquaten Verfügbarkeit zum Wohle der Patienten genutzt werden können (z. B. zur Möglichkeit eines rechtzeitigen Angebots zur Zweitmeinung). Die Vorschläge zur Weiterentwicklung wären kurzfristig (innerhalb von 1-2 Jahren) umsetzbar.

Patients are often unable to accurately classify a healthcare provider's proposed medical treatment so healthcare providers can offer medical treatment beyond the patients' needs. Thus, future-oriented regulation should ensure that the type and volume of observed medical treatment correspond as closely as possible to the actual needs of the patients. Current regulation cannot achieve this goal. A further development of the second opinion can effectively reduce the information asymmetry between healthcare providers and patients. In addition, a further development of the categorisation of DRGs as well as the calculation of their relative weights can significantly reduce unintended financial incentives for healthcare providers. For an informed and future-oriented regulation, it is important for the legislator to ensure that the relevant patientrelated information can be used in Germany for the benefit of the patients with adequate availability. The proposals for further development could be implemented within 1-2 years.

\subsection{Motivation für die Notwendigkeit einer Mengensteuerung}

Bei einer Erkrankung konsultieren Patienten i. d. R. einen Arzt. In diesem Rahmen führt der Leistungserbringer z. B. Untersuchungen durch und stellt zum Abschluss eine Diagnose. Auf Basis dieser Diagnose schlägt der Leistungserbringer dem Patienten dann eine medizinische Behandlung vor, um die mit einer Erkrankung einhergehenden Beschwerden zu lindern bzw. die Erkrankung zu heilen.

Die theoretische gesundheitsökonomische Forschung argumentiert, dass zwischen Patienten und Leistungserbringern allerdings eine Informationsasymmetrie über die Bewertung von medizinischen Sachverhalten besteht (Arrow 1963). Patienten können demnach die Wirkung der vorgeschlagenen medizinischen Behandlung des Leistungserbringers nicht genau einordnen. Unter diesen Umständen können Leistungserbringer die realisierte Menge von medizinischen Behandlungen in ihrem Interesse beeinflussen (Evans 1974 bzw. Pauly 1980). So könnte es z. B. sein, dass Leistungserbringer - zusätzlich zu den Bedürfnissen der Patienten - auch die Vergütung einer medizinischen Behandlung mit in ihre Entscheidung für oder gegen eine medizinische Behandlung einfließen lassen (Ellis und McGuire 1986).

Die empirische gesundheitsökonomische Forschung zeigt, dass die realisierte Menge von medizinischen Behandlungen tatsächlich vom Ausmaß der Informationsasymmetrie und der Vergütung für die Leistungserbringer abhängt. ${ }^{1}$ Die umfassende internationale Evidenz stützt

Neben finanziellen Anreizen können auch nicht-finanzielle Anreize die angebotene Menge von me- 
sich dabei z. B. auf finanzielle Anreize, die von Relativgewichten für Fallpauschalen, Basisfallwerten oder Einkommen ausgehen (siehe u. a. Currie et al. 2011; Dafny 2005; Gruber und Owings 1996; Clemens und Gottlieb 2014 und Afendulis und Kessler 2007). Die Evidenz aus Deutschland bestätigt die internationalen Erkenntnisse (siehe u.a. Schreyögg et al. 2014; Jürges und Köberlein 2015; Bäuml und Dette 2016).

Da die Informationsasymmetrie bzw. die finanziellen Anreize nachweislich ein maßgeblicher Einflussfaktor für die tatsächlich realisierte Menge von medizinischen Behandlungen sind, ist ein regulatorischer Ordnungsrahmen im Sinne einer Mengensteuerung notwendig. Eine effektive Mengensteuerung sollte demnach das Ziel verfolgen, möglichst unmittelbar die durch die Informationsasymmetrie verursachten Abweichungen der Menge von medizinischen Behandlungen zu korrigieren. Eine Mengensteuerung sollte also verhindern, dass über die tatsächlichen Bedürfnisse der Patienten hinaus medizinische Behandlungen mit einer ungewollt hohen Behandlungsintensität (ungewolltes Behandlungsverhalten) oder mit einer fehlenden medizinischen Indikation (ungewolltes Aufnahmeverhalten) erbracht werden.

Ein zusätzliches, allerdings nachrangiges Ziel einer Mengensteuerung kann sein, dass die Krankenkassenbeiträge der Patienten möglichst effizient für die benötigten medizinischen Behandlungen eingesetzt werden. Eine Mengensteuerung zielt dann z. B. zusätzlich auf die durch die Informationsasymmetrie bzw. die finanziellen Anreize verursachten ungewollten Veränderungen in der Dokumentation bzw. der Abrechnung von medizinischen Behandlungen (ungewollte Kodierung) ab. Diese Veränderungen in der Kodierung durch die Leistungserbringer führen dazu, dass sich die beobachtete Menge von medizinischen Behandlungen verändert, ohne dass sich dabei die tatsächlich

dizinischen Behandlungen beeinflussen (z. B. Prestige). realisierte medizinische Behandlung selbst verändert.

Der zweite Abschnitt beschreibt die wesentlichen aktuellen Instrumente der Mengensteuerung in Deutschland und bewertet, inwieweit diese sicherstellen, dass sich die Menge von medizinischen Behandlungen so genau wie möglich an den tatsächlichen Bedürfnissen der $\mathrm{Pa}$ tienten orientiert. Der dritte Abschnitt schlägt mögliche Weiterentwicklungen für eine effektivere Mengensteuerung in Deutschland vor. Im vierten Abschnitt wird ein Fazit gezogen.

\subsection{Aktuelle Instrumente zur Mengensteuerung in Deutschland}

Aus betriebswirtschaftlicher Sicht führt eine Steigerung von Mengen von medizinischen Behandlungen zu einer Senkung von Fixkosten für eine einzelne medizinische Behandlung (sog. positiver Skaleneffekt). ${ }^{2}$ Geringere Fixkosten für eine einzelne medizinische Behandlung führen - bei einer pauschalen Vergütung - zu einem stärker werdenden finanziellen Anreiz zu einer weiteren Steigerung von Mengen von medizinischen Behandlungen (Bäuml und Dette 2016).

Um einer weiteren Steigerung der Mengen von medizinischen Behandlungen durch stärker werdende finanzielle Anreize entgegenzuwirken, hat der Gesetzgeber zwei Instrumente zur Mengensteuerung eingeführt: den Fixkostendegressionsabschlag (FDA) gem. $\$ 4$ Abs. $2 \mathrm{a}$ KHEntgG - neu - sowie die Absenkung von Bewertungsrelationen gem. $₫ 17 \mathrm{~b}$ Abs. 1 S. 5 KHG i. V. m. $₫ 9$ Abs. 1c KHEntgG.

Der FDA ist dabei das hauptsächliche Instrument zur Mengensteuerung in Deutschland. ${ }^{3}$ Leistungserbringer und Krankenkassen

2 Ein positiver Skaleneffekt ist solange zu erwarten, bis zur weiteren Steigerung von Mengen von medizinischen Behandlungen zusätzliche Kapazitäten (z. B. Infrastruktur oder Personal) erforderlich werden.

3 Der FDA bezieht sich auf fast alle medizinischen Behandlungen eines Krankenhauses und sieht nur wenige Ausnahmen vor, z. B. für Transplantationen. Er 
verhandeln i. d. R. jährlich die voraussichtlich notwendige Menge an medizinischen Behandlungen sowie das dafür benötigte Budget. ${ }^{4}$ Durch den FDA wird bundeseinheitlich ein Abschlag in Höhe von 35 \% für eine Dauer von drei Jahren auf das Budget eingeführt, wenn eine Steigerung von Mengen von medizinischen Behandlungen gegenüber dem Vorjahr vereinbart wird.

Der Wirkungsmechanismus des FDA vereitelt allerdings eine der Grundideen, die bei der Einführung der G-DRGs verfolgt wurde: Durch eine pauschalierte Vergütung sollen Leistungserbringer, die eine medizinische Behandlung zu geringeren Kosten als die Wettbewerber erbringen können, diese auch vermehrt erbringen. Weniger effiziente Leistungserbringer sollen diese medizinische Behandlung im Gegenzug dann nicht mehr erbringen, sodass sich die gesamte Menge an medizinischen Behandlungen nicht notwendigerweise verändert. Die Aussicht auf einen höheren Gewinn durch ein Angebot von effizienten medizinischen Behandlungen eines Leistungserbringers steigert damit die Effizienz der Versorgung im Gesamten. ${ }^{5}$

ersetzt den Mehrleistungsabschlag gem. § 4 Abs. 2a KHEntgG - alt - bei dem die Vergütung für die Steigerung von Mengen von medizinischen Behandlungen drei Jahre lang um $25 \%$ gekürzt wurde.

4 Die Aufstellung der Entgelte und Budgetberechnung (AEB) gem. § 11 Abs. 4 KHEntgG bzw. Anlage 1 KHEntgG und der damit verbundene Erlösausgleich gem. § 4 Abs. 3 KHEntgG könnte selbst ebenfalls als Instrument zur Mengensteuerung betrachtet werden. Aus Gründen der Übersichtlichkeit wird der Erlösausgleich in diesem Beitrag nicht näher beleuchtet. Die Erfahrungen aus der Praxis zeigen, dass der Erlösausgleich in der Bewertung als Instrument zur Mengensteuerung ähnlich wie beim FDA bzw. der gezielten Absenkung von Bewertungsrelationen ist. 5 Die Vergütung auf Basis von Fallpauschalen zeigt allen Leistungserbringern eine Benchmark für die üblichen Kosten einer medizinischen Behandlung, an der sich die Leistungserbringer selbst messen können. Leistungserbringer, die eine medizinische Behandlung zu höheren Kosten als die Benchmark erbringen, bekommen sodann einen Anreiz, ihre Kosten zu senken (Shleifer 1985). Die internationale empirische Literatur hat bewiesen, dass DRG-Systeme an sich ein mächtiges Instrument sein könnten,
Der FDA ist als ein eher undifferenziertes Instrument zur Mengensteuerung zu betrachten. Er beeinflusst nebst potenziell unerwünschten Steigerungen von Mengen einer medizinischen Behandlung (d.h. verursacht durch die finanziellen Anreize für die Leistungserbringer) in gleichem Maße auch potenziell erwünschte Steigerungen von Mengen einer medizinischen Behandlung (d. h. verursacht durch tatsächliche medizinische Indikationen). Der FDA stellt damit nicht verlässlich sicher, dass sich die Menge von medizinischen Behandlungen so genau wie möglich an den tatsächlichen Bedürfnissen der Patienten orientiert. Eine für die Erreichung dieses Ziels notwendige Differenzierung zwischen erwünschten bzw. unerwünschten Steigerungen von medizinischen Behandlungen scheint im Rahmen des FDA schwierig.

Als ergänzendes Instrument wurde eine gezielte Absenkung von Bewertungsrelationen eingeführt. Falls es Anhaltspunkte für eine wirtschaftlich begründete Steigerung von Mengen einer medizinischen Behandlung in der Vergangenheit bei mehreren Leistungserbringern gibt, können die dazugehörigen Bewertungsrelationen - i.d. R. gültig für alle Leistungserbringer - durch das Institut für das Entgeltsystem im Krankenhaus (InEK) manuell abgesenkt werden. ${ }^{6}$ Aktuell sind dies hauptsächlich medizinische Behandlungen aus dem Bereich der Rückenmark-, Spinalkanal- und Wirbelsäulen-Operationen (z. B. DRG I10D).

um z.B. medizinisch ungerechtfertigt lange Krankenhausaufenthalte und die damit verbundenen hohen Kosten zu reduzieren (siehe z.B. Coulam und Gaumer 1991; Hodgkin und McGuire 1994; Cutler 1995; Ellis und McGuire 1996). In Deutschland ist nach der Einführung der G-DRGs eine äquivalente Reduktion der Kosten allerdings nicht zu beobachten (Bäuml et al. 2016a).

6 Bestimmte manuelle Absenkungen gelten nicht für alle Leistungserbringer, sondern nur für Leistungserbringer mit einer auffällig großen Menge - gemessen an der Fallzahl eines einzelnen Leistungserbringers im Verhältnis zum Median der Fallzahlen über alle Leistungserbringer hinweg (z. B. DRG 168D, Frakturen am Femur bzw. Erkrankungen der Wirbelsäule). 
Die gezielte Absenkung von Bewertungsrelationen basiert allerdings nicht auf einer empirischen Evidenz gemäß aktueller wissenschaftlicher Standards, sondern ist das Ergebnis von Verhandlungen zwischen Leistungserbringern und Krankenkassen. Aus diesem Grunde ist es fraglich, inwieweit die gezielte Absenkung von Bewertungsrelationen tatsächlich eine effektive Steuerungswirkung hat. Die Liste der betroffenen medizinischen Behandlungen ist wahrscheinlich nicht nur unvollständig, sondern könnte auch gewollte medizinische Behandlungen beinhalten.

Zusätzlich zu den beiden Instrumenten zur Mengensteuerung FDA und gezielte Absenkung von Bewertungsrelationen können noch zwei weitere Instrumente einen wesentlichen Einfluss auf die Menge von medizinischen Behandlungen haben. Zum einen ist dies eine Ex-ante-Kontrolle der angebotenen medizinischen Behandlung (Zweitmeinung), zum anderen die Ex-post-Kontrolle der erbrachten medizinischen Behandlung (Rechnungsprüfung).

Im Rahmen des GKV-Versorgungsstärkungsgesetzes (GKV-VSG) wurde gem. $\$ 27 \mathrm{~b}$ SGB V das Einholen einer Zweitmeinung für bestimmte planbare Operationen ab dem Jahr 2016 festgelegt. Der indikationsstellende Arzt muss den Versicherten mindestens zehn Tage vor einem geplanten Eingriff über das Recht, eine unabhängige ärztliche Zweitmeinung einzuholen, mündlich aufklären. Weitere medizinische Experten können eine weitere Einschätzung über den Gesundheitszustand eines $\mathrm{Pa}$ tienten und den prognostizierten Effekt einer medizinischen Behandlung abgeben. Die somit gewonnene zusätzliche Perspektive kann damit patientenindividuell eine bessere Entscheidungsgrundlage zur Auswahl einer medizinischen Behandlung bieten.

Nachdem bisher lediglich Gebärmutterentfernungen (Hysterektomien) und Mandeloperationen (Tonsillektomie, Tonsillotomie) in der Zweitmeinungsrichtlinie (Zm-RL) des G-BA zu finden sind, ist die ordnungspolitische Wirkung von Zweitmeinungen in Deutschland aktuell noch zu vernachlässigen. Krankenkassenindividuelle ergänzende Angebote können die- se Lücke aktuell noch nicht schließen. Dies liegt vor allem daran, dass Krankenkassen die Patienten in Deutschland noch nicht aktiv und patientenindividualisiert über ihr Angebot informieren dürfen. Zusätzlich erreichen die Informationen zum Gesundheitszustand von $\mathrm{Pa}$ tienten die Krankenkassen für eine Aufbereitung von patientenindividualisierten Angeboten regelmäßig zu spät. Nur ca. $21 \%$ der $\mathrm{Pa}-$ tienten haben länger als eine Woche Zeit zur Entscheidung für eine Behandlung (Zeitspanne von Indikationsstellung bis Krankenhausaufnahme) (De Cruppé und Geraedts 2017).

Eine effektive Rechnungsprüfung kann zumindest dynamisch dazu beitragen, ungewollte finanzielle Anreize zu korrigieren, auch wenn die ungewollte medizinische Behandlung bei Rechnungsprüfung eigentlich bereits erfolgt ist. Leistungserbringer erbringen medizinische $\mathrm{Be}$ handlungen mit einer ungewollt hohen $\mathrm{Be}$ handlungsintensität oder einer tatsächlich fehlenden medizinischen Indikation trotz ungewollter finanzieller Anreize zukünftig nämlich nicht mehr, wenn die ungewollten medizinischen Behandlungen von der Rechnungsprüfung verlässlich also solche erkannt werden. Die aktuelle Konstruktion der Rechnungsprüfung ist allerdings nicht in der Lage, dieses Ziel angemessen zu erreichen.

Das angestrebte MDK-Reformgesetz bringt aus Sicht der Mengensteuerung aber wichtige Weiterentwicklungen der Rechnungsprüfung mit sich. Dies umfasst vor allem zwei Punkte: Erstens soll die Interpretation von medizinischen Sachverhalten in den Deutschen Kodierrichtlinien (DKR) vereinheitlicht werden und damit den Interpretationsspielraum von medizinischen Sachverhalten verringern. Zweitens soll eine Strafzahlung für Leistungserbringer eingeführt werden, wenn eine Abrechnung der Leistungserbringer im Rahmen der Rechnungsprüfung korrigiert werden muss. Fehlende Strafzahlungen verursachen starke finanzielle Anreize für Leistungserbringer, ungewollte bzw. ungewollt intensive medizinische Behandlungen zu steigern und Spielräume bei der Kodierung auszunutzen (Becker 1968; Kölbel 2010). Die Beschränkung der Strafzahlungen 
auf höchstens 10 Prozent des auf Grund der Prüfung durch den Medizinischen Dienst geminderten Abrechnungsbetrages könnte allerdings dazu führen, dass die finanziellen Anreize für eine nicht regelkonforme Abrechnung durch die Leistungserbringer weiterhin zu stark ausgeprägt sind.

Um die Nebenkosten durch die Rechnungsprüfung weiter $\mathrm{zu}$ reduzieren, soll im Rahmen des MDK-Reformgesetzes zudem eine maximale Prüfquote pro Krankenhaus die Anzahl an möglichen Prüfungen durch die Krankenkassen beschränken. Es erscheint aus Systemsicht sinnvoll, der Mengenausweitung von medizinischen Behandlungen durch die Leistungserbringer auf Dauer nicht mit einer Mengenausweitung von Rechnungsprüfungen durch die Krankenkassen zu begegnen. Es könnte allerdings sein, dass diese Beschränkung der Rechnungsprüfung der Krankenkassen die Wahrscheinlichkeit, dass von nicht regelkonforme Abrechnungen aufgedeckt werden, reduziert. Um sicherzustellen, dass die finanziellen Anreize für eine nicht regelkonforme Abrechnung auch bei einer Beschränkung der Rechnungsprüfung weit genug verringert werden, müsste die Strafzahlung beim Aufdecken einer nicht regelkonformen Abrechnung hoch genug sein (siehe z. B. Becker 1968; Shavell 2004).

\subsection{Instrumente für eine effektivere Mengensteuerung}

\subsubsection{Reduktionen der Informationsasymmetrie durch Zweitmeinungs- verfahren}

Die umfassende internationale Evidenz zeigt, dass Zweitmeinungsverfahren die Informationsasymmetrie reduzieren und einer unerwünschten Steigerung von Mengen von medizinischen Behandlungen entgegenwirken können. Aus diesem Grunde können Zweitmei- nungsverfahren ein effektives Instrument zur Steuerung von Mengen medizinischer Behandlungen sein - und dies sogar, bevor diese erbracht werden.

Eine Zweitmeinung hat dazu geführt, dass - je nach medizinischer Indikation - zwischen $2 \%$ (z. B. Hillen et al. 2017) und $69 \%$ (z. B. Ruetters et al. 2016 und Payne et al. 2014) der vom behandelnden Arzt ursprünglich ausgewählten medizinischen Behandlungen angepasst wurden. Die Evidenz aus Deutschland unterstützt die internationalen Erkenntnisse (Kucera 2014). Bei nur ca. 50 \% der Patienten wird die ursprüngliche Diagnose bestätigt. Die zusätzliche medizinische Expertise schlägt z. B. bei Wirbelsäulenoperationen bei ca. $80 \%$ der begutachteten Patienten anstelle der ursprünglichen Operation eine alternative Therapie (z. B. Physiotherapie) vor.

Wichtig ist $\mathrm{zu}$ betonen, dass eine medizinische Behandlung, die nicht den besten Therapieerfolg verspricht oder sogar unnötig ist, die Gesundheitsversorgung der Patienten nicht nur direkt verschlechtern kann, sondern indirekt noch potenziell lebensverändernde $\mathrm{Ri}$ siken mit sich bringen kann. Iatrogene $\mathrm{Be}$ handlungsfolgen (unerwünschte gesundheitliche Folgen einer ärztlichen Behandlung, z. B. aufgrund von Sepsis oder technischen Fehlern) oder inhärente Sterberisiken bei operativen Eingriffen (z. B. ca. 0,6 bis 21 Todesfälle je 100.000 Anästhesie-Patienten; Gottschalk et al. 2011) sollten im Rahmen der Mengensteuerung nicht ignoriert werden.

Die richtige Stellung der Indikation eines Patienten bzw. die Auswahl der für einen $\mathrm{Pa}$ tienten optimalen medizinischen Behandlung ist oftmals nicht einfach von der naturwissenschaftlichen Basis der Medizin ableitbar, sondern basiert stark auf den Erfahrungswerten des einzelnen behandelnden Arztes. Die Diskrepanz in der medizinischen Beurteilung z. B. von bildgebenden Verfahren (25-27 \% von medizinisch überprüften Sachverhalten), histopathologischen Studien (25-37\% von medizinisch überprüften Sachverhalten) oder klinischen Assessments von degenerativen Erkrankungen der Wirbelsäule (35\% von medizi- 
nisch überprüften Sachverhalten) ist nachweislich hoch (Benbassat 2019). In der gesundheitsökonomischen Forschung wird in diesem Kontext oftmals auch von einer „Grauzonen-Medizin“ („grey area medicine“) gesprochen (Cutler 2014).

Bei vielen medizinischen Behandlungsentscheidungen können auch mehr als eine Zweitmeinung wichtig sein. Dies ist vor allem dann der Fall, wenn sich die unterschiedlichen medizinischen Behandlungen über verschiedene Fachgebiete und Sektoren erstrecken, wie z. B. die Orthopädie und die Neurochirurgie bei Bandscheibenvorfällen im Lendenwirbelbereich (Bäuml et al. 2016b).

Zweitmeinungen sind vor allem dann wirkungsvoll, wenn die Einschätzung bzw. die Auswahl der medizinischen Behandlung unabhängig von der Vergütung für die Behandlung selbst erfolgt. Die ausschließlich medizinisch begründete Auswahl der für einen $\mathrm{Pa}-$ tienten optimalen medizinischen Behandlung durch eine oder mehrere Zweitmeinungen reduziert nicht nur die Informationsasymmetrie zwischen dem behandelnden Arzt und dem Patienten, sondern auch eine potenzielle Wissensund Erfahrungslücke zwischen dem behandelnden Arzt und den weiteren medizinischen Experten.

Eine Ausweitung von Zweitmeinungen auch in Deutschland scheint daher vielversprechend. Allerdings binden klassische Zweitmeinungsverfahren nicht unwesentlich finanzielle und personelle Ressourcen. Aufgrund eines sich weiter verschärfenden Ärztemangels in Deutschland, insbesondere in ländlichen Regionen, stellt sich die Frage, inwieweit die personellen ärztlichen Ressourcen für eine Zweitmeinung überhaupt verfügbar sein können.

Um mittels Zweitmeinungen sicherzustellen, dass sich die Menge von medizinischen Behandlungen so genau wie möglich an den tatsächlichen Bedürfnissen der Patienten orientiert, wird eine maschinelle, skalierbare Unterstützung für Ärzte und Patienten notwendig. Eine maschinell erzeugte patientenindividualisierte Zweitmeinung verfügt über einen rei- chen Informationsgehalt. Sie verkörpert nämlich nicht nur eine einzelne Zweitmeinung, sondern aggregiert eine Vielzahl von Zweitmeinungen. Die Anzahl der möglichen maschinell erzeugten Zweitmeinungen entspricht der Anzahl der behandelnden Ärzte in Deutschland oder sogar weltweit, die Patienten mit einem identischen oder sehr ähnlichen Gesundheitszustand behandeln.

Eine maschinell erzeugte patientenindividualisierte Zweitmeinung könnte damit die wahrscheinlichste medizinische Behandlungsentscheidung beinhalten, die eine Abstimmung aller Ärzte über alle Fachbereiche hinweg für einen individuellen Patienten ergeben würde. ${ }^{7}$ Falls die maschinell erzeugte Zweitmeinung von der geplanten Behandlungsentscheidung des einzelnen behandelnden Arztes abweicht, könnte ein klassisches, menschliches Zweitmeinungsverfahren ausgelöst werden (durch krankenkassenabhängige Programme oder aus Akzeptanzgründen besser noch durch krankenkassenunabhängige Institutionen wie z. B. BetterDoc). Auf diese Weise ist eine umfassende Ausweitung von Zweitmeinungen auch in Deutschland möglich, die durch eine konsequente Priorisierung auf die wichtigsten $\mathrm{Pa}$ tienten die finanziellen und personellen Ressourcen schont. - Abb. 9.1 fasst diese Weiterentwicklung der Zweitmeinung zusammen.

Die Technologie zum maschinellen Erlernen bzw. Vorhersagen der optimalen patienten-

Die vergangenen Behandlungsentscheidungen, auf Basis derer die maschinelle Zweitmeinung beruhen würde, könnten im Rahmen einer ersten, etwas einfacheren Entwicklung die Limitation innehaben, dass diese Behandlungsentscheidungen noch durch die Informationsasymmetrie zwischen Leistungserbringer und Patient beeinflusst wurden. Unter der Annahme, dass es ausreichend viele Leistungserbringer gibt, die trotz der Informationsasymmetrie die für den Patienten beste Behandlungsentscheidung treffen, stellt diese Limitation kein Hindernis für eine effektive maschinelle Zweitmeinung dar (Kleinberg et al. 2018). Durch den Einbezug der Behandlungsentscheidungen nach erfolgten klassischen, menschlichen Zweitmeinungsverfahren könnte die maschinelle Zweitmeinung ihre Behandlungsentscheidungen mehr und mehr von der Informationsasymmetrie abstrahieren. 


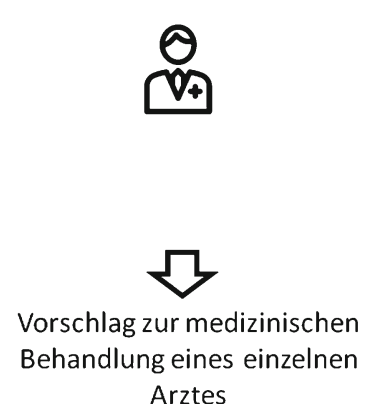

Arztes

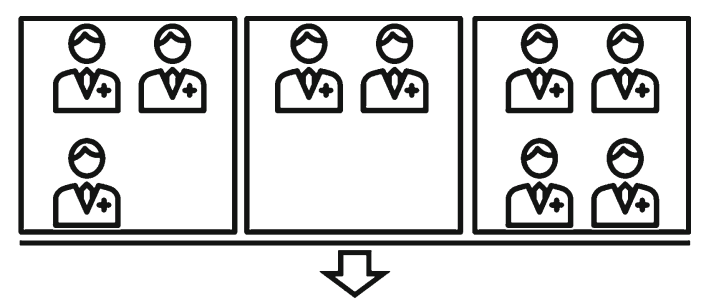

Maschinell erzeugter Vorschlag zur medizinischen Behandlung auf Basis aller $\ddot{\text { Arzte }}$

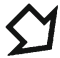

Ist Vorschlag zur medizinischen

Behandlung des einzelnen

Arztes identisch zum maschinell erzeugten Vorschlag?

\section{穴}

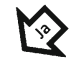 \\ Kein klassisches, \\ menschliches \\ Zweitmeinungsverfahren \\ 回
}

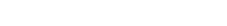

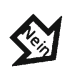

Klassisches, menschliches

Zweitmeinungsverfahren

- Abb. 9.1 Priorisierung von klassischen, menschlichen Zweitmeinungsverfahren mittels maschinell erzeugter patientenindividualisierter Zweitmeinungen

individuellen medizinischen Behandlung auf Basis digitalisierter Informationen zum Gesundheitszustand von Patienten einzelner oder mehrerer Krankenkassen ist bereits verfügbar (Stichwort Künstliche Intelligenz bzw. Maschinelles Lernen). ${ }^{8}$

Um die maschinellen Zweitmeinungen so präzise wie möglich zu gestalten und für so viele Indikationsgebiete wie möglich zu nutzen, ist es wichtig, dass der Gesetzgeber die Verfügbarkeit der aktuell bereits digitalisierten patientenbezogenen Informationen im deutschen Gesundheitswesen verbessert und an internationale Standards anpasst. Die aktuell bereits digitalisierten patientenbezogenen Informationen umfassen z. B. die Abrechnungsdaten der

8 Mehrere Länder haben diese modernen statistischen Methoden auf regulatorischer Ebene bereits eingesetzt und damit wesentliche Verbesserungen in der Patientenversorgung erreicht (z. B. Clalit Research Institute in Israel).
Krankenkassen, Qualitätsdaten aus der sektorenübergreifende Qualitätssicherung (sQS) und weitere klinische Informationen der Leistungserbringer (z. B. Laborwerte, bildgebende Diagnostik, Arztberichte). Aktuell ist der Datenaustausch in Deutschland noch wenig zukunftsorientiert, da z. B. sechs bis neun Monate bei der Übertragung der patientenbezogenen Informationen der niedergelassenen Ärzte bzw. der Kassenärztlichen Vereinigung an die Krankenkassen benötigt werden. Die Zusammenführung der relevanten Informationen z. B. in Form von elektronischen Patientenakten und eine zeitnahe Bereitstellung dieser Informationen für die relevanten Institutionen ist die dringendste und wichtigste Aufgabe für den Gesetzgeber. ${ }^{9}$

9 Die bessere Nutzbarkeit von Gesundheitsdaten ist auch im Rahmen der geplanten Digitalen Versorgung Gesetzes (DVG) geplant. Ob die in diesem Ge- 
Nachdem eine hohe Qualität der maschinellen Zweitmeinung die Patientenversorgung direkt wesentlich verbessern kann, erscheint es aus Sicht der Patienten lohnenswert, die maschinelle Zweitmeinung in einem möglichst großen Wettbewerb entwickeln zu lassen. Die Krankenkassen scheinen für diese Aufgabe gut geeignet zu sein, da sie im Qualitäts- und Preiswettbewerb den Anreiz bekommen würden, die beste maschinelle Zweitmeinung zu entwickeln bzw. entwickeln zu lassen. Kooperationen zwischen Krankenkassen und privaten Anbietern haben bereits ähnliche Produkte innoviert (z. B. die Diagnostik-App von AdaHealth). ${ }^{10}$

In einem weiteren Schritt könnte der Gesetzgeber die Erfassung von patientenbezogenen Ergebnisindikatoren konsequenter umsetzten (z. B. Patient Reported Outcome Measures - PROMs). Die Verknüpfung der Behandlungsentscheidungen mit diesen Ergebnisindikatoren würde zusätzlich eine maschinell erzeugte patientenindividualisierte Zweitmeinung erzeugen, die die Behandlung mit dem besten $\mathrm{zu}$ erwartenden Ergebnis für einen Patienten - auf Basis der Erfahrungen aller identischen bzw. ausreichend ähnlichen Patienten - beinhalten würde. Etablierte patientenbezogene Ergebnisindikatoren wären für viele medizinische Bereiche bereits entwickelt (z. B. International Consortium for Health Outcomes Measurement, ICHOM).

setz angedachten Maßnahmen ausreichen, die Verfügbarkeit und Nutzbarkeit der Daten tatsächlich auf aktuelle internationale Standards anzupassen, ist aktuell noch nicht abschätzbar. Eine Grundlage für die Inhalte könnte dabei der recht einfach implementierbare FIHR-Standard sein. Ein z. B. wöchentlicher Datenaustausch scheint technisch und organisatorisch umsetzbar.

10 Alternativ könnte aber auch der Gesetzgeber selbst einen effizienten Wettbewerb gestalten. Die klassische Ausgestaltung von Vergabeverfahren ist hierfür allerdings ungeeignet. Der Gesetzgeber könnte sich bei der Ausgestaltung aber an den großen Erfolgen von "Preisausschreibungen" anderer, innovativerer Länder bei ähnlichen Herausforderungen orientieren. Ein Beispiel hierfür wäre der "Heritage Health Price" oder die "The SPRINT Data Analysis Challenge" in den USA.

\subsubsection{Reduktionen ungewollter finanzieller Anreize durch systemische Erneuerung der Methode zur Kategorisierung von Fallgruppen}

Neben einer direkten Reduktion der Informationsasymmetrie zwischen Leistungserbringern und Patienten durch Zweitmeinungsverfahren kann zusätzlich eine zielgerichtete Reduktion von ungewollten finanziellen Anreizen unterstützen, dass sich die Menge von medizinischen Behandlungen so genau wie möglich an den tatsächlichen Bedürfnissen der Patienten orientiert.

Ein Ursprung für substanzielle Anreize zur Steigerung von nominalen (ungewolltes Kodierverhalten) und realen (ungewolltes Aufnahme- bzw. Behandlungsverhalten) Mengen von medizinischen Behandlungen liegt im Herzen des Vergütungssystems, dem deutschen DRG-Algorithmus (G-DRG). Das Definitionshandbuch kategorisiert Patienten in klinisch bedeutsame „Produkte“ (diagnosebezogene Fallgruppen, DRGs), für die das InEK patientenspezifische Vergütungen auf Basis historisch beobachteter Kosten festlegt (Bewertungsrelationen).

Damit die patientenspezifischen Vergütungen möglichst präzise sind, nutzt das InEK über die Diagnosen hinaus eine Reihe weiterer Patientenmerkmale zur Kategorisierung der Patienten, wie z. B. Alter, Geschlecht, Prozeduren oder Beatmungsdauer. Zwangsläufig entstehen bei dieser Art der Kategorisierung große diskontinuierliche finanzielle Anreize. Diskontinuierliche finanzielle Anreize zeichnen sich dadurch aus, dass Leistungserbringer für aus medizinischer Sicht ähnliche Patienten sprunghaft unterschiedliche Vergütungen erhalten. Die Vergütung für zwei Patienten, die sowohl identische Diagnosen und Prozeduren als auch ein identisches Alter und Geschlecht aufweisen, z.B. aber um eine Stunde unterschiedlich lange beatmet werden, kann große 
Unterschiede aufweisen. In der Folge könnten DRG-Systeme wie das aktuelle G-DRG-System $\mathrm{zu}$ feingliedrig sein und die Leistungserbringer damit in Abhängigkeit von marginalen Behandlungskosten vergüten anstelle von tatsächlichen Pauschalen. Sie hätten damit wieder eine gewisse Ähnlichkeit zur tagesbezogenen Vergütung in der stationären Versorgung vor der Einführung der G-DRGs in Deutschland (tagesgleiche Pflegesätze für jeden einzelnen Behandlungstag) oder zu der überwiegend nicht gebündelten, einzelleistungsbezogenen Vergütung in der ambulanten Versorgung (Einheitlicher Bewertungsmaßstab) (McClellan 1997; Rosenthal 2007).

Ist die Kategorisierung von Fallgruppen auf diese Art und Weise zu feingliedrig und nach wie vor von marginalen Behandlungskosten abhängig, könnte nicht nur die gewünschte Reduktion der Kosten ausbleiben, sondern die beobachteten Mengen von medizinischen Behandlungen könnten durch eine unerwünscht hohe Behandlungsintensität gekennzeichnet sein (Papanicolas und McGuire 2015; Bäuml und Kümpel 2019). Anstelle von unnötig langen medizinischen Behandlungen, wie sie bei tagesgleichen Pflegesätzen vermutet wurden, könnten also unnötig intensive medizinische Behandlungen die Folge sein. Aus Sicht der ökonomischen Theorie können die unterschiedlichen Vergütungen in Abhängigkeit von den marginalen Behandlungskosten allerdings teilweise auch wünschenswert sein. Falls (v.a. nicht stark auf das Gemeinwohl ausgerichtete) Leistungserbringer jedoch keine nach Behandlungsintensität unterschiedlichen Vergütungen erhielten, könnten diese die Patienten möglicherweise auch unterversorgen (Hafsteinsdottir und Siciliani 2010).

Eine effektive Mengensteuerung scheint aus diesem Grunde schwierig. Verschiedene Maßnahmen im Herzen des G-DRG-Systems gegen ungewollte Steigerungen von nominalen und realen Mengen von medizinischen Behandlungen durch diskontinuierliche finanzielle Anreize sind aber dennoch möglich. So könnte das InEK die im internationalen Vergleich intensive Nutzung von Prozeduren-Kodes (OPS-Ko- des) reduzieren und vermehrt indikationsbasierte Diagnose-Kodes (ICD-Kodes) (z. B. gemäß Leitlinien) soweit wie möglich zur Kategorisierung von Fallgruppen verwenden. Beispiele hierfür wären etwa bei Patienten mit Wirbelgleiten z. B. das Auftreten von muskulären Ausfällen, das Auftreten von Harnverhalt bzw. Stuhlinkontinenz und/oder die Schweregrade nach Mayerding als Konditionen für unterschiedliche Fallpauschalen (z. B. Schmerzbehandlung vs. operative Behandlung). Durch diese Vorgehensweise könnte die Qualität bezüglich der Indikation der Behandlung maßgeblich gefördert werden und die Mengensteuerung darin unterstützen, dass sich die Menge von medizinischen Behandlungen so genau wie möglich an den tatsächlichen Bedürfnissen der Patienten orientiert. Voraussichtlich müsste hierfür der ICD-Katalog um indikationsrelevante Merkmale erweitert werden, was kurzfristig (innerhalb von 1-2 Jahren) umsetzbar wäre. Zusätzlich wäre es förderlich, wenn zur Kategorisierung von Fallgruppen soweit wie möglich nachweisbare Diagnosen oder Patientenmerkmale bzw. Diagnosen mit wenig Interpretationsspielraum verwendet würden. ${ }^{11}$

\subsubsection{Reduktionen ungewollter finanzieller Anreize durch systemische Erneuerung der Methode zur Berechnung der Relativgewichte}

Ein weiterer Ursprung für substanzielle Anreize zur Steigerung von Mengen von medizi-

11 Die zur Klassifizierung genutzten Patientenmerkmale, die durch die Leistungserbringer dokumentiert werden müssen, können i.d. R. weder vom Patienten selbst noch vom Kostenträger perfekt beobachtet werden. Die Leistungserbringer könnten diesen Spielraum in der Dokumentation auch weiterhin systematisch ausnutzen. Ein modernes DRG-System wird damit wahrscheinlich immer unter Ungenauigkeiten in Bezug auf den tatsächlichen Sachverhalt oder die tatsächlich erbrachten Leistungen leiden. 


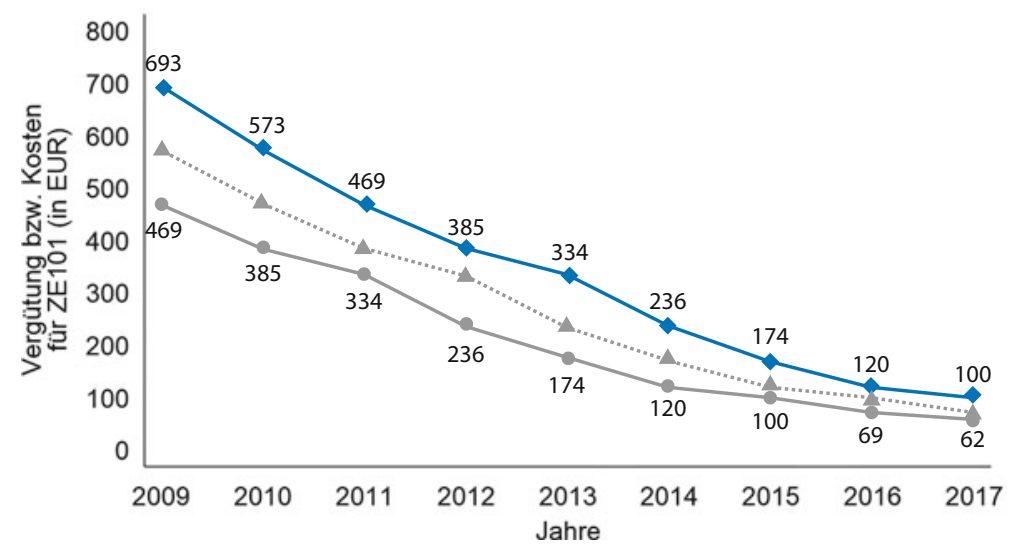

Vergütung für einen Medikamente-

freisetzenden Koronarstent (ZE101)

Tatsächliche Kosten bei Kalkulationslücke $1 \mathrm{Jahr}$

Tatsächliche Kosten bei Kalkulationslücke 2 Jahre

Krankenhaus-Report 2020

- Abb.9.2 Diskrepanz zwischen Vergütung und Kosten für das Zusatzentgelt für einen Medikamente-freisetzenden Koronarstent (ZE101) (Quelle: G-DRG-Fallpauschalen-Kataloge 2009-2019, InEK; eigene Berechnungen)

nischen Behandlungen liegt ebenfalls im Herzen des G-DRG-Systems. Das InEK berechnet jährlich die für alle Krankenhäuser in Deutschland gültigen Relativgewichte auf Basis der von einer Auswahl an Krankenhäusern (Kalkulationskrankenhäuser) zur Verfügung gestellten tatsächlichen Behandlungskosten. Die einem Systemjahr zur Berechnung zugrunde liegenden Behandlungskosten werden allerdings mit einer Verzögerung von zwei Jahren beobachtet.

Es ist nicht unwahrscheinlich, dass sich die tatsächlichen Behandlungskosten in der Zwischenzeit verändern, sodass die für ein Systemjahr berechneten Relativgewichte zum Zeitpunkt der Anwendung nicht mehr aktuell genug sind. Es könnte sich die Produktivität der Krankenhäuser durch eine Optimierung von Prozessen für bestimmte medizinische Behandlungen verbessert haben, so dass Patienten nun z. B. mit weniger Pflegetagen genauso gesund entlassen werden können. Auch könnten sich die Behandlungstechniken verbessert haben, die z. B. die Schnitt-Naht-Zeit im OP-Be- reich, die Eingriffszeit in der endoskopischen Diagnostik bzw. Therapie oder die Behandlungszeit bei therapeutischen Verfahren verkürzen. Durch die Kalkulationslücke von zwei Jahren würden in der Folge finanzielle Anreize zur ungewollten Steigerung für bestimmte medizinische Behandlungen entstehen.

Das nachfolgende Beispiel aus dem Bereich der Sachkosten soll diese Problematik veranschaulichen. Ab dem Systemjahr 2009 führte das InEK bundesweite Zusatzentgelte für Medikamente-freisetzende Koronarstents ein (ZE101). - Abb. 9.2 zeigt auf der y-Achse die Vergütung, die Leistungserbringer für das Einlegen eines Medikamente-freisetzenden Stents in eine Koronararterie (8-837.m0) zusätzlich zur Fallpauschale bekommen (blaue Farbe) sowie die Kosten, die die Leistungserbringer zum gleichen Zeitpunkt tatsächlich für einen $\mathrm{Me}$ dikamente-freisetzenden Stents durchschnittlichen haben (graue Farbe; durchgezogene Linie bei einer vermuteten Kalkulationslücke von zwei Jahren und gestrichelte Linie bei einer vermuteten Kalkulationslücke von einem 
Jahr). ${ }^{12}$ Die x-Achse zeigt die Jahre im Zeitverlauf.

Das Zusatzentgelt für Medikamente-freisetzende Koronarstents bezifferte sich z. B. im Jahr 2013 auf $334 €$. Die tatsächlichen durchschnittlichen Behandlungskosten beliefen sich zum gleichen Zeitpunkt auf vermutete $174 €$ (freiwillig übermittelte durchschnittlichen Kosten aus dem Jahr 2013, die zur Berechnung der Vergütung für das Jahr 2015 maßgebend waren). Die ungewollte Gewinnmarge beträgt im Jahr 2013 somit ca. $92 \%$. Durch die starke Dynamik bei den tatsächlichen Behandlungskosten hat die Verzögerung der Datenverfügbarkeit von zwei Jahren zu einer deutlichen ungewollten Gewinnmarge für die Leistungserbringer von durchschnittlich ca. $30 \%$ (Kalkulationslücke von einem Jahr) bzw. 67\% (Kalkulationslücke von zwei Jahren) geführt. Diese starken finanziellen Anreize können in der Folge dauerhaft eine über die tatsächlichen Bedürfnisse der Patienten hinausgehende Behandlungsintensität bei koronaren Herzkrankheiten hervorrufen - und zwar so lange, bis die Vergütung den tatsächlichen durchschnittlichen Behandlungskosten entspricht.

Im Bereich der Sachkosten hat der Gesetzgeber diese Problematik erkannt und ab dem Jahr 2017 eine Sachkostenkorrektur eingeführt. Die zwischen den Vertragsparteien verhandelte Sachkostenkorrektur auf Basis des $\$ 17 \mathrm{~b}$ Abs. 1 Satz 6 KHG verteilt die Erlöse aller-

12 Die tatsächlichen durchschnittlichen Kosten für Medikamente-freisetzende Stents zum Zeitpunkt der Anwendung der Relativgewichte in einem Systemjahr (z.B. 2013) sind an der Vergütung zwei Jahre nach dem entsprechenden Systemjahr ablesbar (hier folglich 2015). Der Grund hierfür ist, dass für die Berechnung der Vergütung zwei Jahre nach dem entsprechenden Systemjahr (hier folglich 2015) die tatsächlichen durchschnittlichen Kosten von vor zwei Jahren (hier folglich 2013) zur Verfügung gestellt wurden. Zusätzlich hat sich die Definition des Zusatzentgelts nicht verändert, sodass die zugrunde liegende medizinische Behandlung über die Jahre vergleichbar ist. Bei der Berechnung von Zusatzentgelten werden von einem Teil der Krankenhäuser ergänzende Datenlieferungen bereitgestellt, sodass die Kalkulationslücke von zwei Jahre auf ein Jahr verkürzt wird. dings nur pauschal weg von den sachkostenintensiven Fallpauschalen hin zu den nicht sachkostenintensiven Fallpauschalen. Zum einen unterscheidet die Sachkostenkorrektur damit nicht zwischen den einzelnen medizinischen Behandlungen. Die Anteile der Sachkosten werden über alle Fallpauschalen hinweg gleichmäßig abgewertet und können damit die wahrscheinlich unterschiedlichen Trends in den Sachkosten der medizinischen Behandlungen nicht berücksichtigen. Eine Untervergütung für bestimmte sachkostenintensive Fallpauschalen, bei denen sich die tatsächlichen Kosten während der Kalkulationslücke nicht verändern, kann die Folge sein. Zum anderen kommt die durch die Sachkostenkorrektur transparent gewordene Übervergütung nicht den Beitragszahlern zugute, sondern wird dazu genutzt, eine Übervergütung für die nicht sachkostenintensiven Fallpauschalen zu erzeugen. Eine zielgerichtete Reduktion von Fehlanreizen wird mit der aktuellen Sachkostenkorrektur nicht erreicht.

Mit der Nutzung von mehr als nur dem letzten verfügbaren Datenjahr an Behandlungskosten zur Berechnung der Relativgewichte könnten z. B. zeitliche Trends im Rahmen der Berechnung geschätzt und die Vergütungen dementsprechend angepasst werden. Durch eine Weiterentwicklung der statistischen Methoden, die bei der Berechnung der Relativgewichte eingesetzt werden, können die finanziellen Anreize zur ungewollten Steigerung von medizinischen Behandlungen damit zielgerichtet und umfassend reduziert werden.

Zudem wäre es sinnvoll, bei der Berechnung der Relativgewichte moderne Konzepte aus dem Bereich des maschinellen Lernens einfließen zu lassen. Ein Beispiel hierfür wäre, dass die Beurteilung der Güte der G-DRGs (und damit für die Kategorisierung der Fallpauschalen und die Berechnung der Relativgewichte) nicht innerhalb der zur Berechnung der Relativgewichte genutzten Daten zu Behandlungskosten erfolgt (sog. In-sample-Beurteilung), sondern auf Basis von nicht genutzten, z. B. möglichst aktuellen Daten zu Behandlungskosten (sog. Out-of-Sample-Beurteilung). Nicht ge- 
nutzte Daten zu Behandlungskosten könnten zum Beispiel das letzte Quartal des Datenjahres sein oder noch besser aus einer Zwischenlieferung bzw. einer erweiterten Nachlieferung der Krankenhäuser aus dem ersten Quartal des Jahres der tatsächlichen Kalkulation kommen. Auf diese Weise würde die Über- bzw. Untervergütung (nicht nur bedingt durch Veränderungen bei den Sachkosten) transparent werden.

\subsection{Fazit}

Informationsasymmetrie über die Bewertung medizinischer Sachverhalte zwischen Leistungserbringern und Patienten macht es möglich, dass Leistungserbringer über die Bedürfnisse der Patienten hinaus medizinische Behandlungen anbieten. Aus diesem Grunde sollte ein regulatorischer Ordnungsrahmen im Sinne einer Mengensteuerung sicherstellen, dass sich die Art und Menge von medizinischen Behandlungen so genau wie möglich an den Bedürfnissen der Patienten orientieren. Die Instrumente der aktuellen Mengensteuerung können dieses Ziel nicht erreichen.

Dieser Beitrag schlägt drei Weiterentwicklungen der aktuellen Mengensteuerung vor, die kurzfristig (innerhalb von 1-2 Jahren) umsetzbar wären:

1. Umfassende Ausweitung von Zweitmeinungen, die direkt die Informationsasymmetrie zwischen Leistungserbringern und Patienten effektiv reduziert. Sobald es der Gesetzgeber unterstützt, sollten Krankenkassen in diesem Rahmen moderne statistische Verfahren anwenden, die eine zielgerichtete Auswahl der für ein Zweitmeinungsverfahren wichtigsten Patienten möglich machen und damit die finanziellen und personellen Ressourcen schonen.

2. Weiterentwicklung der Kategorisierung der Fallgruppen, die ungewollt sprunghafte finanzielle Anreize im Rahmen des bestehenden G-DRGs zielgerichtet mindert.

3. Weiterentwicklung der Berechnung der Relativgewichte, die ungewollt über- bzw. untervergütete Fallpauschalen im Rahmen des bestehenden G-DRGs zielgerichtet reduziert.

Für die Umsetzung der Ausweitung der Zweitmeinungen müsste der Gesetzgeber allerdings die Verfügbarkeit zumindest der aktuell bereits im deutschen Gesundheitswesen verfügbaren und digitalisierten patientenbezogenen Informationen verbessern. Diese Vorarbeit ist die dringendste und wichtigste Aufgabe für den Gesetzgeber, um eine zielgerichtete und zukunftssichere Regulierung bzw. Steuerung zum Wohle der Patienten auch in Deutschland zu ermöglichen.

Eine effektive Steuerung der Mengen von medizinischen Behandlungen hängt allerdings stets auch mit der Steuerung der Kapazitäten zusammen. Nicht nur aus diesem Grunde scheint eine andauernde organisatorische Trennung der Steuerung der Mengen (Mengensteuerung durch Selbstverwaltung) und der Steuerung der Kapazitäten (Bedarfsanalyse im Rahmen der Krankenhausplanung durch Bundesländer) wenig zukunftsorientiert (Gerlach et al. 2018). Wie die Erfahrungen im Rahmen des GKV-Gesundheitsreformgesetzes 2000 zeigen, scheint eine Umstellung der dualistischen Krankenhausfinanzierung auf Monistik kurzfristig allerdings nicht umsetzbar zu sein. Die Überführung der Steuerung der Kapazitäten in die Selbstverwaltung wird eine mittel- bis langfristige Herausforderung für die Gesetzgebung sein.

Die in der politischen Diskussion öfters erwähnten Instrumente zur Mengensteuerung (siehe z. B. Augurzky et al. 2012) wie z. B. Regionalbudgets (sog. Preislösung, ähnlich wie der FDA) oder Zertifikatehandel (sog. Mengenlösung) sind nicht geeignet, um das wesentliche Ziel einer zielgerichteten und zukunftsorientierten Mengensteuerung zu erfüllen. ${ }^{13}$ Beide

13 Regionalbudgets sehen i. d. R. eine gemeinsame sektorübergreifende Vergütung für Krankenhäuser und niedergelassene Ärzte und auf Basis von festen Jahresbudgets mit unterjährigen Abschlagszahlungen vor. Krankenhäuser können ungewollte medizinische Behandlungen vermeiden, sodass der Preis pro medizinischer Behandlung steigt, oder weiterhin er- 
Instrumente zielen vor allem darauf $a b$, dass die Krankenkassenbeiträge der Patienten möglichst effizient eingesetzt werden. Sie können aber nicht sicherstellen, dass vor allem medizinische Behandlungen mit einer ungewollt hohen Behandlungsintensität oder mit einer fehlenden medizinischen Indikation vermieden werden. ${ }^{14}$

\section{Literatur}

Afendulis CC, Kessler DP (2007) Tradeoffs from integrating diagnosis and treatment in markets for health care. Am Econ Rev 97:1013-1020

Arrow KJ (1963) Uncertainty and the welfare economics of medical care. Am Econ Rev 53:941-973

Augurzky B, Felder S, Gülker R, Mennicken R, Meyer S, Wasem J (2012) Mengenentwicklung und Mengensteuerung stationärer Leistungen. Gutachten im Auftrag des GKV-Spitzenverbands. Essen/Basel, Duisburg

Bäuml M, Dette TC (2016) G-DRG side effects: hospital responses to Germany's inpatient reimbursement system. In: Dette TC (Hrsg) Essays in applied micro economics. Doctoral dissertation. Harvard University

Bäuml M, Kümpel C (2019) Hospital responses to the introduction of reimbursements by treatment intensi-

bringen, sodass der Preis pro medizinischer Behandlung fällt (im Sinne einer sog. Pigou-Steuer mit nichtlinearen Eigenschaften). Die Menge von medizinische Behandlungen ist dabei nicht beschränkt.

14 Darüber hinaus ist eine Verbesserung der Mengensteuerung mittels Zertifikate-Handel aufgrund der typischen Eigenschaften des Krankenhausmarktes in Deutschland nur schwer sinnvoll implementierbar. Die Grundidee bei einem Zertifikate-Handel ist, dass diejenigen Marktteilnehmer, die am einfachsten und am günstigsten die gesamthaft begrenzte Menge reduzieren können (hier: Leistungserbringer mit negativen oder niedrigen Deckungsbeiträgen), diese auch am stärksten reduzieren. Dieser Mechanismus funktioniert aber aus theoretischer Sicht i.d. R. nur, wenn die Marktteilnehmer im betrachteten Markt in einem (möglichst) perfekten Wettbewerb zueinander stehen. Aufgrund der sehr regional organisierten Krankenhausmärkte müsste für jeden regionalen Krankenhausmarkt (z. B. Kreisebene) ein eigener Zertifikate-Handel etabliert werden. Andernfalls könnte eine Mengensteuerung mittels Zertifikate-Handel die durchschnittliche Indikationsqualität sogar weiter verschlechtern und die Versorgungssicherheit gefährden. ty in a (presumably lump sum) DRG system. In: Kümpel C (Hrsg) Essays on health economics - effects of reimbursement incentives on hospitals and on providers in long-term care. Doctoral dissertation. Universität Hamburg, Hamburg

Bäuml M, Kifmann M, Kümpel C (2016a) Ökonomische Trends - Entwicklung des Krankenhausmarktes. Wirtschaftsdienst 12:932-934

Bäuml M, Kifmann M, Krämer J, Schreyögg J (2016b) Bandscheibenoperationen - Patientenerfahrungen, Indikationsqualität und Notfallkodierung. In: Böcken J, Braun B, Meierjürgen R (Hrsg) Gesundheitsmonitor 2016 Bürgerorientierung im Gesundheitswesen. Bertelsmann Stiftung, Gütersloh

Becker GS (1968) Crime and punishment: an economic approach. J Polit Econ 76:169-217

Benbassat J (2019) Obtaining a second opinion is a neglected source of health care inequalities. Isr J Health Policy Res 8:12

Clemens J, Gottlieb JD (2014) Do physicians' financial incentives affect medical treatment and patient health? Am Econ Rev 104:1320-1349

Coulam RF, Gaumer GL (1991) Medicare's prospective payment system: a critical appraisal. Health Care Financ Rev 13:45-77

De Cruppé W, Geraedts M (2017) Hospital choice in Germany from the patient's perspective: a crosssectional study. BMC Health Serv Res 17:720

Currie J, Lin W, Zhang W (2011) Patient knowledge and antibiotic abuse: evidence from an audit study in China. J Health Econ 30:933-949

Cutler DM (1995) The incidence of adverse medical outcomes under prospective payment. Econometrica 63:29-50

Cutler DM (2014) The quality cure: how focusing on health care quality can save your live and lower spending too. University of California Press, Berkeley, Los Angeles

Dafny LS (2005) How do hospitals respond to price changes? Am Econ Rev 95:1525-1547

Ellis RP, McGuire TG (1986) Provider behavior under prospective reimbursement: cost sharing and supply. J Health Econ 5:129-151

Ellis RP, McGuire TG (1996) Hospital response to prospective payment: moral hazard, selection, and practicestyle effects. J Health Econ 15:257-277

Evans RG (1974) Supplier-induced demand: some empirical evidence and implications. In: Perlman M (Hrsg.) The economics of health and medical care. Macmillan, London, S 162-173

Gerlach F, Greiner W, Haubitz M, Meyer G, Schreyögg J, Thürmann P, Wille E (2018) Finanzierung sicher, Überkapazitäten abbauen. In: Sachverständigenrat zur Begutachtung der Entwicklung im Gesundheitswesen (Hrsg) Gutachten 2018: Bedarfsgerechte Steuerung der Gesundheitsversorgung. Sach- 
verständigenrat zur Begutachtung der Entwicklung im Gesundheitswesen, Bonn/Berlin

Gottschalk A, Van Aken H, Zenz M, Standl T (2011) Is anesthesia dangerous? Dtsch Arztebl 108:469-474

Gruber J, Owings M (1996) Physician financial incentives and cesarean section delivery. RAND J Econ 27:99123

Hafsteinsdottir EJG, Siciliani L (2010) DRG prospective payment systems: refine or not refine? Health Econ 19:1226-1239

Hillen MA, Medendorpa NM, Daamsb JG, Smets EMA (2017) Patient-driven second opinions in oncology: a systematic review. Oncologist 22:1197-1211

Hodgkin D, McGuire TG (1994) Payment levels and hospital response to prospective payment. J Health Econ 13:1-29

Jürges H, Köberlein J (2015) What explains DRG upcoding in neonatology? The roles of financial incentives and infant health. J Health Econ 43:13-26

Kleinberg J, Lakkaraju H, Leskovec J, Ludwig J, Mullainathan S (2018) Human decisions and machine predictions. Q J Econ 133:237-293

Kölbel R (2010) Die Prüfung der Abrechnungen von Krankenhausleistungen in der Gesetzlichen Krankenversicherung - Eine Bewertung aus kriminologischer Perspektive. Gutachten für den AOK-Bundesverband, Bielefeld

Kucera M (2014) Jedem zweiten Patienten wird von OP abgeraten - Zweitmeinungen. kma 19:8
McClellan M (1997) Hospital reimbursement incentives: an empirical analysis. J Econ Manag Strategy 6:91128

Papanicolas I, McGuire A (2015) Do financial incentives trump clinical guidance? Hip replacement in England and Scotland. J Health Econ 44:25-36

Pauly M (1980) Physician information and the consumer's demand for care. In: Pauly M (Hrsg) Doctors and their workshops: economic models of physician behavior. National Bureau of Economic Research, University of Chicago Press, Chicago, S 43-64

Payne VL, Singh H, Meyer AND, Levy L, Harrison D, Graber ML (2014) Patient-initiated second opinions: systematic review of characteristics and impact on diagnosis, treatment, and satisfaction. Mayo Clin Proc 89:687-696

Rosenthal MB (2007) Nonpayment for performance? Medicare's new reimbursement rule. $\mathrm{N}$ Engl J Med 357:1573-1575

Ruetters D, Keinki C, Schroth S, Liebl P, Huebner J (2016) Is there evidence for a better health care for cancer patients after a second opinion? A systematic review. J Cancer Res Clin Oncol 142:1521-1528

Schreyögg J, Bäuml M, Krämer J, Dette T, Busse R, Geissler A (2014) Endbericht Forschungsauftrag zur Mengenentwicklung nach $\S 17 \mathrm{~b}$ Abs. $9 \mathrm{KHG}$. In: EK

Shavell S (2004) Foundations of economic analysis of law, 1. Aufl. Harvard University Press, Cambridge

Shleifer A (1985) A theory of yardstick competition. Rand J Econ 3:319-327

Open Access Dieses Kapitel wird unter der Creative Commons Namensnennung 4.0 International Lizenz (http:// creativecommons.org/licenses/by/4.0/deed.de) veröffentlicht, welche die Nutzung, Vervielfältigung, Bearbeitung, Verbreitung und Wiedergabe in jeglichem Medium und Format erlaubt, sofern Sie den/die ursprünglichen Autor(en) und die Quelle ordnungsgemäß nennen, einen Link zur Creative Commons Lizenz beifügen und angeben, ob Änderungen vorgenommen wurden.

Die in diesem Kapitel enthaltenen Bilder und sonstiges Drittmaterial unterliegen ebenfalls der genannten Creative Commons Lizenz, sofern sich aus der Abbildungslegende nichts anderes ergibt. Sofern das betreffende Material nicht unter der genannten Creative Commons Lizenz steht und die betreffende Handlung nicht nach gesetzlichen Vorschriften erlaubt ist, ist für die oben aufgeführten Weiterverwendungen des Materials die Einwilligung des jeweiligen Rechteinhabers einzuholen. 